\title{
Devorando el Hallyu: Desarrollo, hibridación y canibalismo latinoamericano
}

\section{Devouring the Hallyu: Development, Hybridization, and Latin American Cannibalism}

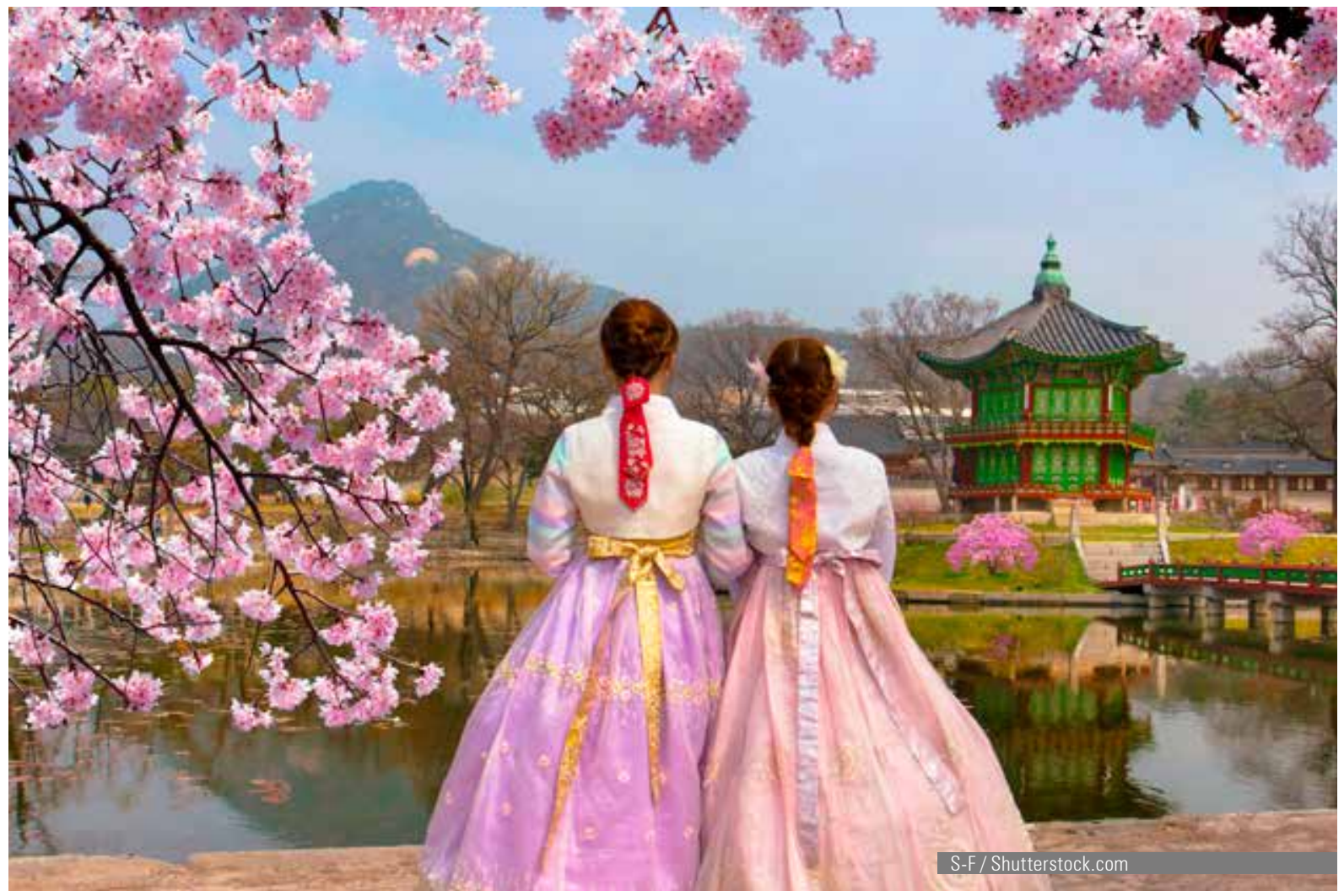

\section{$>>$ Claudio Arriojas}

Universidad Católica Andrés Bello quiet77@gmail.com

Indira Valentina Réquiz

Escuela de Idiomas Modernos de la Universidad Central de Venezuela valentinarequiz@gmail.com

Recibido: 26 de marzo 2019

Aprobado: 25 de abril 2019

Publicado: 28 de junio 2019

\section{Resumen}

El presente artículo desarrolla una breve historia de la ola cultural coreana o Hallyu, sus generaciones y fisonomía. De igual modo, se analiza el crecimiento de este fenómeno en Asia y en América Latina; a partir del paralelismo entre la asimilación de las tecnologías, modos de representación Occidental de la industria creativa coreana y el diseño de políticas públicas por parte del Estado coreano. Finalmente, se discute el carácter híbrido del Hallyu, el carácter totalizador de la etiqueta "cultura coreana" de este, así como la necesidad de abordar esta manifestación desde de un constructo cultural propio de América Latina; para ello, se utiliza la propuesta de antropofagia cultural. 


\section{Palabras clave:}

Hallyu, cultura, Corea, antropofagia, Latinoamérica

\section{Abstract}

This article presents a brief history of the Korean wave or Hallyu, its generations and physiognomy. It also analyses the growth of this phenomenon in Asia and Latin America based on the parallelism between the assimilation of technologies, Western modes of representation in the Korean creative industry, and the design of public policies by the Korean State. Finally, the hybrid character of the Hallyu will be discussed as well as the totalizing character of the label "Korean culture", and also the need to approach this manifestation from a cultural construct specific to Latin America; for this, the cultural anthropophagy proposal is used.

\section{Keywords}

Hallyu, culture, Korea, anthropophagy, Latin America

\section{Introducción}

El Hallyu (한류) u Ola Coreana es un fenómeno cultural registrado desde el final de los años 90, consiste en la producción, consumo y exportación de producciones musicales, visuales y audiovisuales de la República de Corea hacia otros países del este de Asia en primer momento, abarcando luego el resto del mundo.

En un principio, el Hallyu se desarrolló en la esfera privada de la economía. Sin embargo, después este fue incentivado por el Estado y así, "ha sido clave en la promoción y difusión de la imagen de Corea en muchos países alrededor del mundo". (Lopez Rocha, 2015:171).

El término Hallyu se registra según Valadez (2016) a finales del año 2000 en el diario chino The People's Daily que, como parte de un reportaje sobre este fenómeno, compone el término a partir de las palabras Han - Coreay lyu —río—, consolidándose a nivel mediático hasta ser aceptada globalmente. De este modo, se muestra cómo la palabra que define a la cultura pop coreana es un préstamo chino.

\section{Razones del éxito en Asia}

Hogarth describe nueve razones que explican el éxito del Hallyu en China y el resto de Asia. En primer lugar, enumera la alta calidad con relación a la competencia, "Sofisticadas tomas de cámara y ángulos" (Hogarth, 2013:137); en segundo lugar, la belleza fotográfica y una "cinematografía impresionante, lugares escénicos en estaciones cambiantes, edificios interesantes, actores y actrices jóvenes y atractivos, moda y maquillaje magníficos, espléndidos trajes..." (lbíd); en tercer lugar, el ethos asiático, donde el público se identifica con un el reparto debido a su fisonomía y en cuyo discurso encuentra

(una) filosofía de vida y el sistema de valores...orientados hacia el confucianismo, como el centrismo familiar, la piedad filial, el respeto por los ancianos, la lealtad, el amor entre hermanos, el énfasis en la educación, etcétera (Ibíd).

En cuarto lugar, el concepto asiático de lo predestinado como línea de la historia, así como la actitud de los creadores hacía el sexo:

La única sugerencia de sexo entre los amantes es el nacimiento de su bebé, generalmente después del matrimonio. Esta modestia (...) contribuyó inicialmente 
al éxito (...) en China y su difusión a otros países asiáticos y más tarde al mundo islámico. (Ibíd: 137-138).

En quinto lugar, la nueva imagen del hombre, la mujer y su rol social que,

(...) aunque siguen siendo modestas, las mujeres son retratadas como personas fuertes, independientes, capaces, de altos principios, con el valor de su convicción, que superan las dificultades incluso en circunstancias extremas. (lbíd: 138).

En sexto lugar, la dicotomía entre tradición y modernidad, que se explica como "El respeto por la cultura tradicional coreana tanto como la nueva cultura globalizada orientada al Western, resuena entre los espectadores asiáticos". (Ibíd: 139). En séptimo lugar el bajo costo, en tanto "inicialmente se podían obtener en China por una fracción de los precios de los productos japoneses..." (Ibíd:139); en octavo lugar, la habilidad de la narración, que captura al espectador a partir de su sencillez y que estos

Evitan temas controvertidos, como la homosexualidad, el abuso y la prostitución, y tratan sobre el amor eterno puro entre un hombre y una mujer -jóvenes y solteros-, el triángulo amoroso, el altruismo, la familia, la amistad, la humanidad (Ibíd:139);

y por último, las temáticas universales en torno a los ideales de verdad, bondad y belleza.

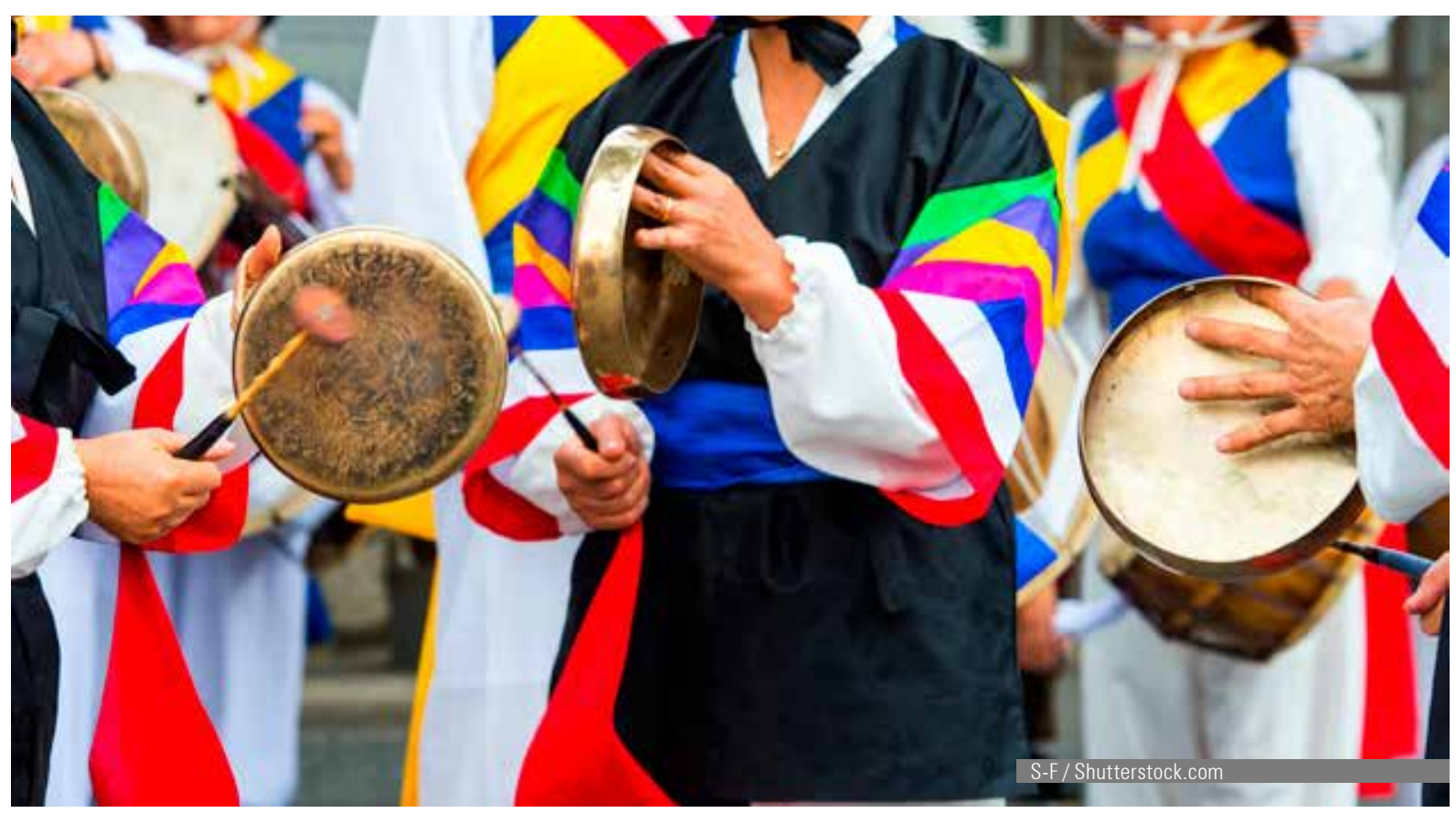

\section{Recuento histórico}

Ahora bien, ¿de dónde proviene este fenómeno? Las producciones que se circunscriben al Hallyu han abandonado casi siempre los métodos de representación tradicionales, al asumir arquetipos de la representación occidental desde una óptica propia, actuar que deriva del proceso de "occidentalización" de Corea a partir del fin de la guerra y la relación económico militar con Estados Unidos. Por ejemplo, "Corea comenzó a 'importar' el sistema legal de EE.UU. y Japón después de la liberación en 1945" (Ryzhkov, 2014: 3). 
Posada (2013), citando a García-Blanch, clasificará tres fases del desarrollo tecnológico coreano, donde la primera será la imitación; la segunda, la adquisición de licencias y transferencias tecnológicas extranjeras; para finalmente apuntar a la innovación. Estas fases fueron la base para el crecimiento de la industria ligera y pesada en el renacimiento económico coreano y será la misma receta que se aplicará con las industrias creativas.

Por otro lado, Sung (2010) afirma que, durante la década de 1980, el Estado coreano recurre a una política de promoción de la cultura popular sobre la cultura Occidental que a la fecha había desplazado a la primera; estas políticas tuvieron resultados en poco tiempo, porque aumentaron el consumo doméstico de productos culturales coreanos y sentaron las bases de las industrias creativas que lo sucederán; así,

A principios de la década de 1990, el gobierno coreano eliminó las restricciones a la participación de los chaebols en las industrias cinematográfica, musical y de radiodifusión. Como resultado, las grandes empresas -entre ellas Samsung, Lotte, Hyundai, LG y SK- entraron en múltiples sectores de la industria cinematográfica y de la radiodifusión mediante la adquisición de varias empresas importantes, como empresas de gestión, de producción y de distribución. (Berg, 2015:2003)

Estos conglomerados no inyectarán grandes cantidades de capital, sino que, corporativizarán la industria del entretenimiento, al darle valores y estructuras que respondan a una lógica comercial; finalmente, terminaron retirándose de la industria musical y cinematográfica como consecuencia de la crisis económica asiática de 1997; lo que permitió, sin querer, un funcionamiento de mercado occidental dentro de estas industrias, en las cuales "el capital de riesgo y las empresas de inversión se convirtieron en los principales financiadores de la producción cinematográfica local” (Shim, 2011:214), con el consiguiente abandono de la óptica del 'arte por el arte' en pos de la recuperación de la inversión y la respuesta a las necesidades del mercado.

Un artículo de Straits Times de 20013 —Citado por Sung (2010) - sugiere que los dramas coreanos en la época de la crisis asiática tenían costos hasta $75 \%$ más baratos que sus pares japoneses y hasta $90 \%$ más baratos que las de Hong Kong, decantando en la continua exposición del televidente del este de Asia a producciones coreanas y generando finalmente gusto.

(...) Lili, una productora de la estación de GTV en Taiwán, dijo que la principal razón por la que la estación había estado transmitiendo telenovelas coreanas era su calidad y precio, en comparación con la calidad y el precio de productos japoneses comparables: Las telenovelas coreanas eran de mejor calidad que los productos locales taiwaneses, pero más baratas que otros productos extranjeros. (Ibid:30)

Estas producciones eran dobladas al idioma del país donde se transmitía y, además de las variables económicas que serán fundamentales para entender la expansión de del consumo de los dramas en la región, se explica también, según Schulze (2013), por un sentimiento compartido de lo asiático que permitirá al televidente encontrarse en elementos confucianos y valores compartidos, como la piedad y el respeto por los ancianos.

Los profundos cambios institucionales en el sector coreano del entretenimiento durante los noventa, así como la influencia de los modos de producción y representación occidentales se explican como una coevolución de la industria que permite "explicar y analizar la dinámica de las industrias creativas, ya que trata de la relación e interacción entre una industria y su entorno institucional"(Berg, 2015:1994). La explosión geométrica que tuvo esta con la masificación del internet y las plataformas de entretenimiento, cimentó la industria nacional de entrenamiento y la impulsó de tal modo, que Corea es, según Shim (2011), el único país post 
guerra de Vietnam donde los espectadores volvieron a preferir producciones nacionales en detrimento de Hollywood.

\section{La participación del Estado}

De acuerdo a Sung (2010), Berg (Berg, 2015), Ryzhkov y López (2017), el Estado coreano decidió tener mayor participación dentro de la industria del entrenamiento a través de impulsos como la Ley de Promoción de la Industria Cultural Básica —1999-, la creación de la Agencia Coreana de Cultura y Contenidos — 1999_; la definición del Hallyu como poder blando del Estado con miras a la región; la creación de la Agencia de Cultura y Contenidos en 2001; la participación institucional del Ministerio de Cultura, Deportes y Turismo, a través de la KOCCA — especializada en radio, animación y videojuegos - y la KOFIC —especializada en el financiamiento de la industria cinematográfica y que cuenta con un fondo de alrededor de 430 millones de dólares- así como del Consejo Nacional de Marcas; la convocatoria a las empresas del sector a mantener conversaciones en 2005 con las instituciones del Estado para el reimpulso del Hallyu y la creación, en 2009, de la Comisión Presidencial de la Marca País. Como se puede ver, el Estado tejió un ecosistema institucional de apoyo y respaldo a la producción de las principales industrias creativas.

El apoyo del gobierno coreano es visible también a través de los órganos de Estado Nacional y embajadas. La construcción de una Marca País y los resultados en la industria turística han creado una "cultura puente" entre el modo de vida Occidental y la tradicionalidad del pueblo coreano, quien no solo exporta entretenimiento sino formas de vida y de entender el mundo, con ficciones y realidades particulares, que logran su cometido; pues exportan a toda una nación. Cheng (2008) — citado por Ryzhkov y López- afirmará que

El incremento en los esfuerzos de marca país en Corea sugería que la representación diplomática evolucionara y que el intercambio cultural y las exportaciones debían ser considerados como una parte integral del cambio político de Corea del Sur" (Ryzhkov y López, 2017:10).

Ejemplo de ello, la creación del Centro Cultural Coreano en América Latina, así como el apoyo a la creación de programas académicos para los estudios coreanos.

En este marco, el Estado coreano abre el mercado latinoamericano al vincular a las instituciones académicas del país en el extranjero con el Hallyu,

En este marco se inscribe la difusión de las industrias culturales coreanas hacia América Latina, el financiamiento de Centros de Estudios Coreanos, de clases de idioma en diversos institutos y universidades y el establecimiento de Centros Culturales en nuestra región.

Paralelamente, la estrategia gubernamental se enfocó en la difusión de una selección de telenovelas cuyos derechos fueron cedidos sin costo alguno a varias cadenas televisivas latinoamericanas y que lograron una notable aceptación en un público lejano, tanto en términos geográficos como culturales; y la conformación de comunidades de seguidores que, animados por el producto de una industria cultural, expendieron sus inquietudes a otras áreas de la cultura propiamente coreana, y abrieron una forma particular de comunicación intercultural. (ladevito y Bavoleo, 2015:32)

Lo anterior redefinió la extensión del Hallyu, en principio limitado a los dramas y más tarde al K-pop; a partir de 2006 y como respuesta a una baja en el rating, paso a describir la industria audiovisual, videojuegos, K-pop y animaciones, esto como una estrategia gubernamental por cimentar su poder blando, mostrando un primer interés por el mercado latinoamericano. Finalmente 
de acuerdo a Bae (2012), citado por Ryzhkov, "el gobierno coreano ha puesto en marcha el K-Culture Promotion Task Force, que tiene como objetivo ayudar a iniciar la era del 'Hallyu 3.0', fijando su objetivo inicial en el 'desarrollo creativo de la cultura tradicional'" (Ryzhkov, 2014:3).

¿De qué se trata el Hallyu 3.0? se integran a los productos culturales el resto de las industrias creativas, como la belleza y el turismo, así como el Han Style; se trata de la difusión y exportación de la cultura tradicional de Corea, a saber,

El Ministerio de Cultura, Deportes y Turismo ha tomado la iniciativa con su nueva campaña de promoción, Han Style, que se centra en seis grandes aspectos de la cultura tradicional coreana: han'gul 한글 —alfabeto coreano-, hansik 한식 — festival de comida fría en abril—, hanbok 한복 — vestido tradicional coreano-, hanok 한옥 — casas tradicionales coreanas-, hanji 한지 — periódico tradicional coreano—y han'guk ǔmak 한국음악 —música tradicional coreana—. (FinchumSung, 2009:47).

Esto podrá evidenciarse en las exposiciones y actividades culturales que las embajadas de Corea han organizado en la región latinoamericana.

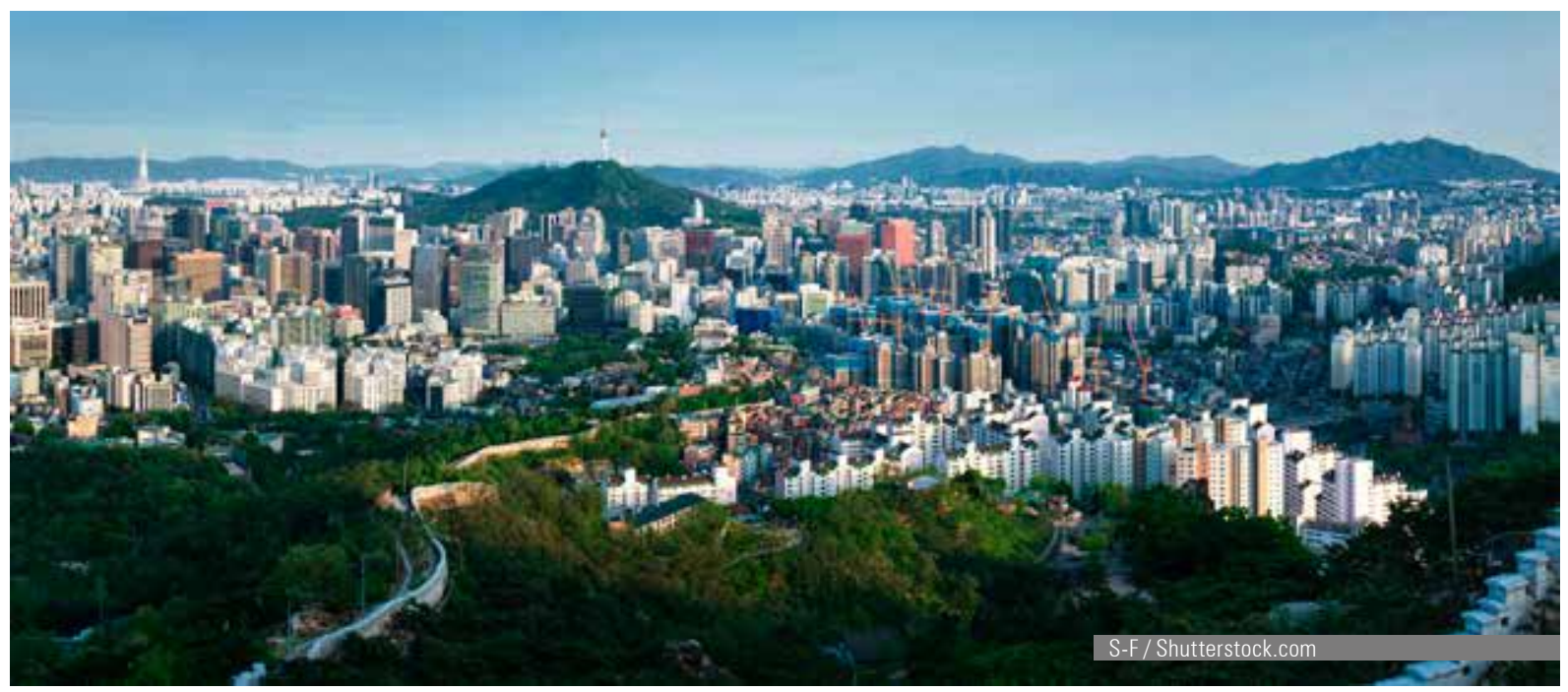

\section{Hallyu en América Latina}

La incursión de los productos coreanos en América Latina rompe con la argumentación de cercanía cultural que leímos hasta ahora en diversos autores; de igual modo se trata de un mercado acostumbrado a consumir productos estadounidenses de altísima calidad técnica y narrativa a precios relativamente bajos, vista la tendencia regional a la piratería y la particular difusión del Hallyu a través de sitios web gratuitos administrados por fans. Finalmente, afirmando lo obvio, no se encontrarán puentes idiomáticos, históricos o culturales entre ambas culturas.

Para entender lo arriba mencionado, Bodomo y Chabal (2014) proponen la Teoría de la Construcción de Identidad Intercultural a Larga Distancia —en inglés LDCCICT-, donde el consumidor global define y se relaciona con otras identidades culturales a partir del consumo de sus productos culturales, sin ser necesario vínculo territorial o personal alguno, más allá del simple gusto. A partir del "continuo entrelazamiento mundial de los grupos culturales, podemos anticipar un aumento constante en la prominencia tanto de las identidades biculturales como de las múltiples identidades" (Smith, 2011. La 
identidad bicultural es a priori un concepto discutible en un entorno globalizado; ya que, más allá de casos específicos de familias multiétnicas o binacionales donde la exposición continua a dos culturas resulta determinante, esta reduce sus posibilidades en los casos de asimilación de valores culturales por una exposición digital a distancia.

El concepto de pluriculturalidad, y más aún, de múltiples identidades culturales conviviendo dentro de un mismo consumidor, permite comprender una de las dinámicas culturales fundamentales del siglo XXI, sobre todo cuando se trata de una cultura que históricamente se ha comportado herméticamente. Yoon (2015) menciona el soonhyuljui o pureza étnica, en la cual la tradición coreana abrazaba el concepto de sangre y de pureza racial como parte de la definición de lo coreano. El proceso de occidentalización de Corea es históricamente reciente y mucho más rápido que los tiempos generacionales, por lo que aún conviven aspectos de estas tradiciones en las generaciones mayores. A diferencia de Occidente, cuya tradición tiene setenta años, Corea adoptó el multiculturalismo dentro de sus políticas culturales recién en el año 2009, "Fue la primera vez que Corea reconoció seriamente a otras culturas y buscó formas de coexistir con ellas". (Yoon, 2015:423).

Ahora bien, en este proceso de convergencia entre las culturas latinoamericanas y la coreana, es válido preguntarnos si el consumidor latino realmente consume productos culturales coreanos puros; "las telenovelas coreanas han penetrado y se han mixturado con las distintas culturas populares latinoamericanas, y en este proceso de relocalización dejan de 'existir'" (ladevito \& Bavoleo, 2015:35).

Finchum-Sung afirma que los consumidores de K-Dramas entienden el producto consumido como perteneciente a "una cultura específica con características culturales claramente decodificables. Pero un análisis detallado y exhaustivo de los blogs y tablones de anuncios de los aficionados internacionales revela que tal cosa es insostenible" (Finchum-Sung, 2009: 392) Pp. 392).

Así, la adecuación de las formas de producción de las industrias creativas coreanas a los requerimientos y estilos del mercado internacional permiten la discusión sobre dicha 'pureza'; donde el Hallyu asume nuevas características del mercado. Shuling Huang, asevera que "La industria de la televisión coreana copió el género de dramas de moda de Japón, produciendo espectáculos (...) para atraer a la afluente generación joven llamada shinsedae". (Ryzhkov y López Rocha, 2017:11).

A su vez Hogarth clasifica los dramas en dos categorías,

Melodrama romántico y ficción histórica. Los que pertenecen a los primeros recuerdan a los tear jerkers de Hollywood de los años 50, y los guiones son similares a las populares novelas victorianas v/escritas por Dickens, Hardy y las Hermanas Bronte". (Hogarth, 2013:140)

Por otro lado, ladevito y Bavoleo (2015) nos presentan el término Hallyuwood, como la fusión entre Hallyu y las formas de producción de Hollywood, mientras Che-gyu (2011) plantea que el Planet Hallyuwoodk es una creación que siempre se calculó a través de las fronteras, es decir, con una mirada puesta en el éxito extranjero de las industrias culturales coreanas, por sobre el consumidor doméstico.

\section{¿Es el Hallyu coreano?}

Ho (2012), citado por Hogarth (2013), plantea que el K-pop recibió una influencia Occidental superior a los dramas de televisión, en tanto los últimos reproducen valores culturales típicos de Corea mientras que el K-pop incorpora modos de representación estadounidenses y bailes de influencia afrocaribeña, al utilizar movimientos de caderas y de piernas, por sobre los de 
hombros y brazos que son comunes a la danza tradicional coreana.

Sin embargo, Hogarth afirma que

Para cualquiera que haya asistido a un ritual chamánico Icut, no es sorpresivo que Corea produzca TAN -mayúsculas del autor- talentosas estrellas del pop. La cultura dionisíaca, es decir, el vino, el canto y la danza, siempre ha sido parte integrante de la vida coreana (Hogarth, 2013: 143-144)

Así, no hay consenso entre los autores sobre la naturaleza del Hallyu como industrias creativas típicamente coreanas; incluso existe discusión cuando se generaliza la cultura 'popular' como un predio del Hallyu, sobre esto tenemos que:

La cultura popular como un ente único no existe, tal como se manifiesta comúnmente en Corea. Las culturas populares, en plural, son todas aquéllas que se crean en la sociedad por todos los integrantes de estas, no solo por un grupo que maneja una imagen específica de una sociedad y su cultura, y que la promueve como la única expresión de cultura popular de su sociedad. (López Rocha, 2015: 177).

Las industrias relacionadas con el Hallyu oligopolizaron, hasta el impulso del Han Style, la visión de lo popular de la cultura coreana, transformándose en el único paso de comunicación entre el consumidor extranjero y lo que suponemos es lo "coreano". Esto significa que, aunque se han utilizado las herramientas de la globalización para la difusión de los productos culturales, Hwy-Chang y Parc (2013) plantean que la industria privada coreana posee una estructura oligopólica, hecho que se evidencia en la existencia de grandes corporaciones que controlan la práctica totalidad del mercado, mientras desarrollan iniciativas con miras a controlar toda la cadena productiva dentro de su área de acción. Por ejemplo, las llamadas fábricas de idols. No podemos dejar de mencionar que esta forma de funcionamiento es muy similar al del "tradicional" chaebol.

Shim (2011), afirma que Herbert Schiller revisa su tesis del imperialismo cultural estadunidense post guerra fría, para explicar que la globalización cultural se traduce en la instrumentalización de conocimientos técnicos, formas de gerencia, producción y comercialización de Estados Unidos, a través de corporaciones transnacionales no necesariamente americanas.

Orlik (2009), citado por (Ko, Kim, No, \& Gobbi, 2014), propone que los dramas coreanos llevan al espectador a una realidad ajena al individuo; es decir falseable. El Hallyu termina describiendo "un mundo imaginado creado a través de la participación de escritores, directores, actores y espectadores de K-Dramas" (Schulze, 2013:377-378).

Sin embargo, la globalización y el internet ya han permitido en otros sectores y sociedades el surgimiento de emprendimientos, artistas y producciones que no se encuentran vinculados a las grandes corporaciones. Aún más, resulta inocente aceptar que el proceso de creación de los productores de Hallyu no se encuentra repleto de significados y referencias de la cultura en que nació, creció y se desarrolló el productor.

A pesar de esto, Fan (2006) citado por Ryzhkov y López Rocha (2017), planteará que "la marca país que está siendo promovida, podría parecer "extranjera" para la audiencia local" (Ryzhkov \& López Rocha, 2017:22),

La mayoría de los jóvenes coreanos — que participaron en el estudio- piensan que el Hallyu no es un elemento que represente correctamente a su sociedad ni a su cultura en el exterior, evidenciando así la inexistencia de alguna correspondencia identitaria con este fenómeno cultural que tan popularmente ha sido diseminado en el mundo poniendo en los imaginarios extranjeros el nombre de Corea. (Ibíd: 25). 
Resulta interesante realizar una revisión del análisis de resultados de este estudio, ya que la mayoría de los encuestados parece entender conceptos similares por Hallyu, K-pop, y no los referentes culturales que plantea el Hallyu 3.0 y el Han Style, claro, estos conceptos responden a políticas dirigidas al extranjero; otra posibilidad es la no clasificación de lo cotidiano como cultural por parte del nativo.

En las producciones coreanas

Los jóvenes e intérpretes combinan su experiencia en la interpretación tradicional con sonidos y ritmos de baile producidos electrónicamente, y la imagen que se presenta al público es la de una cultura juvenil coreana genial y consciente. (Finchum-Sung, 2009:1392).

Esto sin duda repercute en la apreciación que tiene el espectador nacional sobre el producto cultural que consume, ya que, si bien este procede de una tradición artística nacional, incorpora junto a la tecnología cultural occidental, formas de representación exógenas que sin embargo, no tienen que ser vistas como un abandono de la cultura nacional.

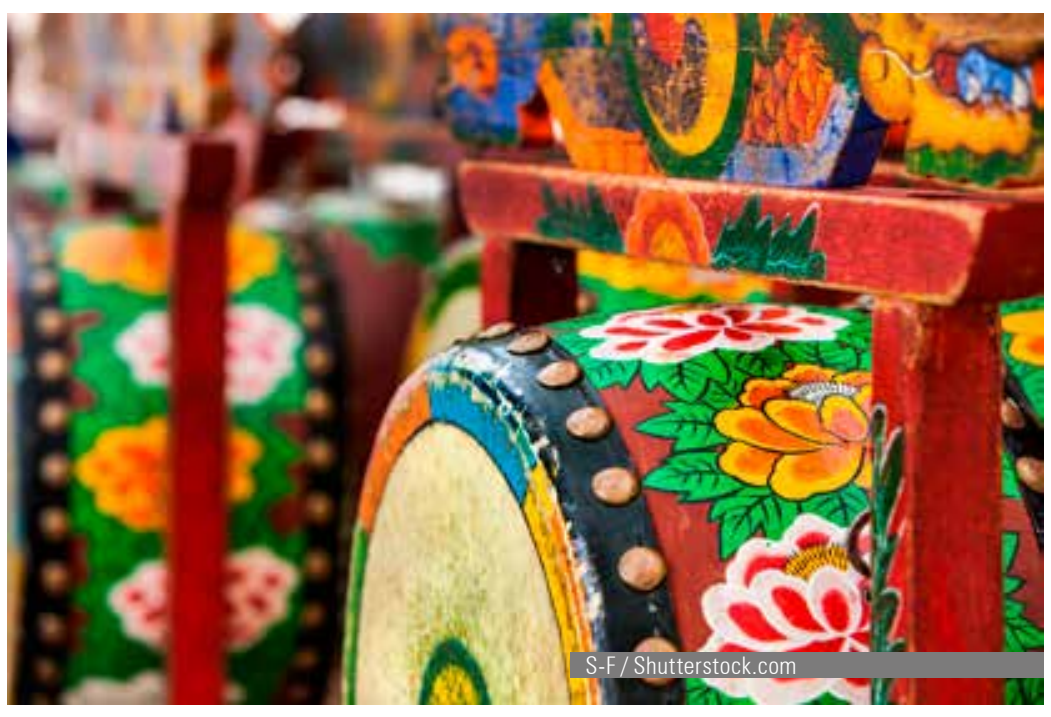

En el caso de las telenovelas, "la circulación y el consumo de las telenovelas coreanas 'crean' un espacio/canal para la comunicación social intercultural entre Corea y —en nuestro caso- los países latinoamericanos" (ladevito \& Bavoleo, 2015:27). Este espacio de comunicación ha ido incorporando las políticas públicas anunciadas por el Estado coreano al punto de producir, por ejemplo, dramas y largometrajes del subgénero zombie, en ambientes históricos como la unificación de los tres reinos de Corea; lo que aporta a una historia de interés comercial contemporáneo, el idioma, trajes, vituallas, arquitectura e incluso personajes históricos que serán finalmente digeridos por el consumidor latinoamericano.

Hu y Wu afirman que el "argumento clave de la función de utilidad del consumidor, que considera que los bienes culturales y no culturales son complementarios entre sí". (Huh y Wu, 2017:1392). Es decir, que el consumidor no tiene necesariamente que distinguir el carácter cultural o no de un bien cuando lo consume, sino que los encuentra complementarios, y por ende, indisolubles de su propia experiencia de consumo. Es así, como sectores fundamentalmente juveniles de Latinoamérica se han convertido en consumidores de cultura coreana, a la par de la tecnología. Por ejemplo, juzgando la calidad de ambas y encontrando la manifestación cultural cercana así, por los 
valores compartidos occidentales; pero a la vez interesante por sus medios de producción, historias exóticas para el latino y además, porque la producción cultural se encuentra particularmente dirigida a este consumidor.

La búsqueda objetiva de un grado de pureza cultural resulta por definición ingenuo, las culturas se desarrollan en una relación de continuo intercambio con su entorno natural y humano, por ello, no es posible mantener en un plano práctico alguna integridad originaria.

(Se) toma la coreanidad o cultura coreana tradicional y moderna, en forma de autenticidad y adopta elementos de la cultura global para configurarse, propagarse e interactuar con otras culturas y sociedades del mundo. (...) el Hallyu se considera híbrido porque contiene elementos locales-coreanos y elementos extra locales no coreanos en su composición. (Lopez Rocha, 2015, Pp. 179).

\section{Hibridez cultural}

Por híbrido, de acuerdo a Kim y Yang (2006), Ryou (2008) y Shim (2004) citados por Yoon, se entiende "una mezcla cultural de influencias occidentales. Los productos culturales exportados a Asia en nombre de la ola coreana deben ser vistos como variaciones de la cultura o híbrido occidental " (Yoon, 2015:426), no como un producto exclusivamente coreano.

El concepto de hibridación cultural será desarrollado por el argentino Néstor García Canclini en el libro Culturas híbridas, fundamental para los estudios culturales como disciplina, que más tarde comienza a utilizarse como unidad de análisis de los investigadores interesados en los estudios coreanos.

García realizó una redefinición de acuerdo a la discusión epistemológica posterior a la publicación del libro, definiendo hibridación como los "procesos socioculturales en los que estructuras o prácticas discretas, que existían en forma separada, se combinan para generar nuevas estructuras, objetos y prácticas". (García Canclini, 2003:3).

Así, podemos entender en un primer momento al Hallyu como una hibridación entre diferentes culturas asiáticas y el modo de producción Occidental; como se ha visto con anterioridad, las prácticas económicas que definieron al Hallyu provienen de la historia económica contemporánea de Corea, en su relación de interdependencia con las naciones limítrofes, mientras que se gestaba un proceso similar con la cultura Occidental, particularmente la estadounidense.

Retondar (2008) afirma que el constructo "hibridación cultural" resulta particularmente útil cuando se estudia el espacio de interrelación entre dos esferas sociales, a modo de estructura interna y externa. Ninguno de estos procesos cultural es 100\% homogéneo, así, el espacio de interrelación del Hallyu, no se da particularmente con los Estados Unidos, los países de Asia del Este o con Latinoamérica, sino que también se manifiesta entre la generación joven y adulta de Corea, el campo y la ciudad, la capital y la provincia, y es por tanto un proceso de continua evolución.

Más tarde, García Canclini afirma que:

Estos procesos incesantes, variados, de hibridación llevan a relativizar la noción de identidad. Cuestionan, incluso, la tendencia antropológica y de un sector de los estudios culturales a considerar las identidades como objeto de investigación. El énfasis en la hibridación no solo clausura la pretensión de establecer identidades "puras" o "auténticas". Además, pone en evidencia el riesgo de delimitar identidades locales autocontenidas, o que intenten afirmarse como radicalmente opuestas a la sociedad nacional o la globalización (García Canclini, 2003:5)

Así, debemos partir de la inexistencia de una cultura coreana "pura" en contraposición a los productos culturales de las industrias creativas corea- 
nas. La identidad como área de investigación se difumina, toda vez que debe ser reconocida como un contenido en constante adaptación y mutación.

Las prácticas tradicionales de la cultura coreana se encuentran muy bien delimitadas por lo tardía de su apertura a la comunidad global. Sin embargo, estas respondían a lógicas económicas y sociales que no se corresponden con las posibilidades de la nación coreana en la actualidad. Por ende, como parte del proceso de transformación de la identidad nacional, estas están destinadas a transformarse.

El Hallyu es un producto cultural industrialmente elaborado de la mano del Estado, oligopolios empresariales y el mercado en su acepción de mano invisible y por ende, el consumidor latinoamericano de Hallyu, habituado al consumo de estas producciones, corre el riesgo de transformarse en gourmets multiculturales, "viajar entre patrimonios y saborear sus diferencias. Los patrimonios históricos, entendidos de este modo abierto y cambiante, pueden enriquecerse y actuar como puentes de comprensión entre sociedades distintas". (García Canclini, 1999: Parr. 9) y así, el Hallyu es una oportunidad.

\section{Antropofagia coreana}

Se-Woong Koo (2014) afirma que durante la dinastía Joseon el Estado legisló el canibalismo infantil intrafamiliar donde, invocando la "piedad filial" los niños podían voluntariamente ofrecer sangre o un dedo a sus padres moribundos, quienes sanaban de forma milagrosa.

Existen registros del siglo XVI de la obtención forzosa de hígados y vesículas biliares como una cura para la lepra, que más tarde serían consideradas cura para las enfermedades venéreas por miembros de la corte, lo que significó la erradicación de los mendigos y el asesinato de niños.

Lo que estos dos tipos de canibalismo compartían era la creencia en el poder de las partes del cuerpo humano para sanar. Se creía que la vesícula biliar curaba la lepra, y la sangre y la carne de los niños podían despertar a los padres moribundos. La objeción de las autoridades al canibalismo, si es que existe, es por la violencia cometida contra personas involuntarias cuyos órganos y carne fueron recolectados, y por la violencia contra la sociedad cuyos valores morales se ven amenazados por la obtención y el consumo no aprobados de partes humanas. Pero la convicción en el poder medicinal del cuerpo humano nunca fue cuestionada. (Se-Woong, 2014: Parr. 8-9)

Finalmente Se-Woong menciona un manual policial de supersticiones que clasifica en 1926 el canibalismo como cura para la lepra y la exhumación de cadáveres de niños como remedio universal.

\section{Canibalismo}

La forma de hibridación coreana puede abordarse desde otra categoría, la del canibalismo caraiba que planteó Oswald de Andrade en su Manifiesto Antropófago (1981). Se trata del manifiesto fundacional de la antropofagia cultural y del modernismo literario brasileño, y como su nombre lo indica, es una declaración de principios y no un artículo académico.

En un primer acercamiento al manifiesto, Rolnik afirma que los modernistas

(...) criticaban las políticas del deseo y de la creación cultural vigente. Con humor cáustico, apuntaron especialmente a los intelectuales académicos, que se doblegaban de manera patética ante la cultura dominante y posaban, con arrogancia, de portadores de la verdad. (Rolnik, 2011:8) 
Sin embargo, De Andrade logro impulsar una discusión estética entre los modernistas brasileños de la época, calando en los estudios culturales, apuntando a la antropofagia cultural como unidad de análisis.

Se considera que la antropofagia cultural es una unidad de análisis válida para estudiar la cultura pop coreana, vista la necesidad de abordar el Hallyu como una manifestación cultural de Latinoamérica desvestida, "lo que obstaculizaba la verdad era la ropa, el impermeable entre el mundo interior y el mundo exterior" (Andrade, 1981:67).

Así, se entiende la antropofagia como un acto de evolución cultural, en tanto,

Fundada sobre una alteridad constitutiva, el antropófago en tanto tal, contiene en sí siempre un Otro, deconstruye todos los tropos que en el presente postulan las políticas identitarias y particularistas. El antropófago de Oswald emerge amenazante porque, precisamente, postula una mezcla irreductible. La antropofagia desactiva cualquier idea de universalismo, porque no postula una Otredad absoluta. (Cámara, 2013:26)

De esta manera, el modernismo permitió plantear el abandono de la vieja tradición teórica del conquistado, siempre en desventaja técnica y cultural con el conquistador, para dar lugar a una propuesta donde el primero sobrevivía y es persé, un ser creador de cultura, capaz de generar una unidad de análisis y una cultura cuyo nacimiento es únicamente latinoamericano y fundamentalmente ritual.

(...) la antropofagia como ritual estaría hablando de la manera de percibir el fenómeno artístico en América Latina, relacionado con la aproximación conscientemente a este en un acto de elevado espíritu, con el propósito de nutrirse de la proteína canónica que rige los parámetros occidentales del arte, bajo la perspectiva de algunos estudios estéticos sobre los que se funda su historia (Martínez, 2005:257)

La antropofagia cultural se basa en el mito de algunas tribus indígenas del caribe brasileño las cuales creían que, a partir de la digestión física del enemigo se asimilaba la fortaleza y las virtudes de este; situación que se repite con sus bemoles en otras tribus de ascendencia caribe hasta la actualidad, como es el caso de la tribu Yanomami en Venezuela, cuyo ritual funerario consiste en la cremación y deglución de las cenizas de sus familiares muertos, no abandonándolos en la tierra en caso de una migración. "De la apropiación física a la apropiación simbólica del otro cuerpo hay, apenas, un pequeño paso y de la alimentación de la gestualidad señalizadora del alimento el trayecto es bastante corto y lógico". (Baitello, 2004:164)

Latinoamérica es una comunidad que aún se pregunta por su ser cultural, sin encontrar posible respuesta en cada una de las partes integrantes. Más allá de la definición de una identidad propia, a partir de la diferencia.

El canibalismo plantea, que a partir de la Conquista española de Latinoamérica se da una canibalización del colonizador y su cultura, así mismo, como plantea Nirschack, "Los objetos de la digestión antropofágica son, por supuesto, todo el patrimonio cultural de los poderes hegemónicos, sus conocimientos y sus ciencias, pero también sus tecnologías y sus invenciones técnicas". (Nitschack, 2016:161). 


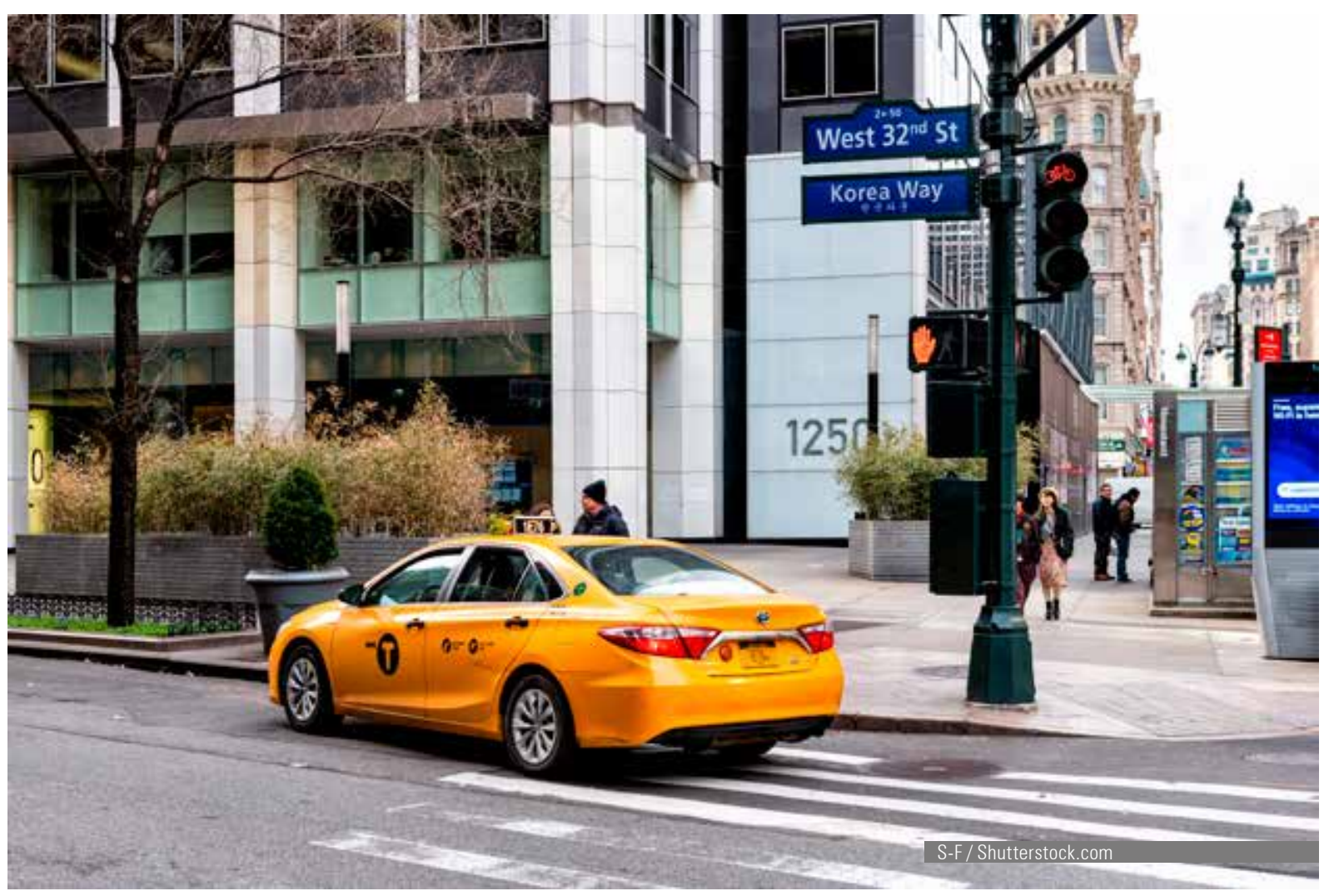

\section{Conclusión}

El canibalismo crea un ser cultural nuevo, relacionado con lo autóctono y lo exógeno, pero único. Así, la asimilación de las fortalezas de la otredad no implica una continua ganancia, sino que también consiste en renunciar a lo que se era, sin que esto implique una desaparición total. De esta forma, la cultura imperial romana no solo pervive en los idiomas romances y en el derecho continental sino también en usos, costumbres, tradiciones y creencias, que en su seno se gestaron.

Cuando aplicamos esta visión a "la forma de producción de la cultura pop coreana termina siendo una mezcla de elementos locales y extra-locales, se ha reinventado a través de numerosos procesos que cada vez le han dado nuevas características". (Ryzhkov, Andrii, 2014, Pp. 9). Estos procesos son la canibalización de Occidente, de Asia y de la propia cultura coreana.

Andrade creía que "la apropiación de las tecnologías más avanzadas no iban a poner en cuestión la identidad brasileña" (Nitschack, 2016:169) y como hemos asistido hasta ahora, tampoco la de Corea.

La antropofagia, si bien no conceptualmente, es una alegoría que puede explicar el desarrollo cultural de la humanidad a partir del continuo intercambio y asimilación de valores culturales y tecnologías que garantizan la supervivencia y una creciente calidad de vida para el ser humano.

Nitschack plantea que la "antropofagia no sería solamente la práctica cultural con la cual el subalterno mantiene su identidad frente a las culturas hegemónicas, antropofagia sería también el procedimiento de la renovación cultural con validez para toda la humanidad". (2016:160). La antropofagia permitió a Corea imprimir a sus producciones patrones de calidad técnicos 
que definieron su futura mundialización, y con ello, el país difundió los valores tradicionales de una cultura milenaria, así como la riqueza del idioma coreano y el alfabeto Hangul, pieza fundamental en la difusión de la cultura coreana. En tanto la lengua es el principal generador de cultura del hombre como especie.

La apropiación del espacio y sus recursos, la apropiación del tiempo y sus atributos, la apropiación de las mentes y sus imágenes no siempre pasan por la relación directa de apropiación entre dos cuerpos... entonces, con esto, tenemos el surgimiento de la inocofagía. (Baitello, 2004:164)

Es posible entender el proceso de digestión de la cultura coreana pop imagen - y tradicional, a partir de la unidad de análisis planteada por Oswald de Andrade. La historia de América Latina y de Corea tienen algunas similitudes: ambos fuimos continuamente diezmados y conquistados; ambos vivimos a la sombra de grandes potencias; ambos tuvimos canibalismo literal, cultural y ambos estudiamos al otro a través de los estudios coreanos y latinoamericanos; ante el futuro quedan preguntas por responder, ¿Qué caníbal consumirá al otro?

\section{Referencias bibliográficas}

- Andrade, Oswald de (1981): Obra escogida, Caracas, Biblioteca Ayacucho.

- Baitello Junior, N. (2004). Las cuatro devoraciones. Iconofagia y antropofagia en la comunicación y la cultura. Revista Internacional de Comunicación Audiovisual, Publicidad y Literatura, 1 (2), 159-168. Recuperado de: https://idus.us.es/xmlui/bitstream/handle/11441/57556/las_cuatro_devoraciones_ iconofagia_y_antropofagia_en_la_comunicacion_y_la_cultura.pdf?sequence $=1$ fisAllowed $=y$

- Berg, S. (2015). Creative Cluster Evolution: The Case of the Film and TV Industries in Seoul, South Korea. European Planning Studies, 23(10), 1993-2008. Recuperado de: https://doi.org/10.1080/09654313.201 4.946645

- Bodomo, A., \& Chabal, E. S. (2014). Africa-Asia relations through the prism of television drama: How Africans in Hong Kong Conceptualize Korean Culture. African and Asian Studies, 13(4), 504-529. Recuperado de: https://doi.org/10.1163/15692108-12341319

- Cámara, M. (2013) Utopía y barbarie, la operación aqntropofágica de Oswald de Andrade. Ipotesi, 17; 1; 7-2013; 17-27. Recuperado de: http://hdl.handle.net/11336/3790

- Che-gyu, K. (2011). Planet Hallyuwood:, 14(1), 197-212.

- Finchum-Sung, H. (2009). Image is everything: re-imaging traditional music in the era of the Korean wave. Southeast Review of Asian Studies, 31 (1), 39-55.

- García Canclini, N. (2003). Noticias recientes sobre la hibridación. Trans. Revista Transcultural de Música, 7, 1-17. Recuperado de: https://www.redalyc.org/pdf/822/82200702.pdf

- García Canclini, N. (5 de 12 de 1999). Gourmets multiculturales. Recuperado de La Jornada Semanal: https://www.jornada.com.mx/1999/12/05/sem-nestor.html

- Hogarth, H. K. K. (2013). The Korean wave: An Asian reaction to western-dominated globalization. Perspectives on Global Development and Technology, 12(1-2), 135-151. Recuperado de: https://doi. org/10.1163/15691497-12341247

- Huh, C. G., \& Wu, J. (2017). Do Hallyu (Korean Wave) Exports Promote Korea's Consumer Goods Exports? Emerging Markets Finance and Trade, 53(6), 1388-1404. Recuperado de: https://doi.org/10.1080/15404 96X.2017.1313161

- Hwy-Chang, M., \& Parc, J. (2013). Korean dramas and films: Key factors for their international competitiveness. Asian Journal of Social Science, 41(2), 126-149. Recuperado de: https://doi. org/10.1163/15685314-12341295

- Iadevito, P., \& Bavoleo, B. (2015). Korean soap operas in Latin America a cultural studies approach. Revista Digital Mundo Asia Pacífico, 4(6), 25-39. Recuperado de: https://doi.org/10.17230/map.v4.i6.02

- Ko, N., Kim, J.-N., No, S., \& Gobbi, R. (2014). The Korean Wave Hallyu in Looking at Escapism in Peruvian Society. Perspectives on Global Development and Technology, 13, 332-346. Recuperado de: https://doi. org/10.1163/15691497-12341305

- Lopez Rocha, N. (2015). El rol del Hallyu como cultura pop en la creación y la difusión de la imagen de la mujer coreana contemporánea. http://www.portesasiapacifico.com.mx/?P=articulogid=307, 9(18), 171-196. Recuperado de: http://dspace.uan.mx:8080/xmlui/handle/123456789/186

- Martínez D. (2005) Antropofagia: Hábito y ritual en América Latina Cultural. AISTHESIS No 38.251-265. 
Recuperado de: http://revistaaisthesis.uc.cl/index.php/rait/article/view/512/481

- Nitschack, H. (2016). Antropofagia cultural y tecnología. UNIVERSUM, 31(2), 157-171. Recuperado de: https://scielo.conicyt.cl/pdf/universum/v31n2/art 10.pdf

- Posada Suárez, C. (2013). De reino ermitaño a tigre asiático. El modelo de desarrollo de Corea del Sur. Revista Digital Mundo Asia Pacífico, 2(3), 6-14. Recuperado de: http://publicaciones.eafit.edu.co/index. $\mathrm{php} / \mathrm{map} /$ article/view/2210/2126

- Retondar, A. (2008). Hibridismo cultural: ¿clave analítica para la comprensión de la modernización latinoamericana? La perspectiva de Néstor García Canclini 1. Sociología (Méx), 23(67), 33-49. Recuperado de: http://www.scielo.org.mx/pdf/soc/v23n67/v23n67a3.pdf

- Roknik, S. (2011). antropofagia zombie. En WV.AA., Umbrales. Fugas de la institución total: entre captura y vida (págs. 1-25). Sevilla: Universidad Internacional de Andalucía. Recuperado de: http://ayp.unia.es/ dmdocuments/umbrales21.pdf

- Ryzhkov, A. (2014). TRENDS FOR INTERNATIONALIZATION OF KOREA: From K-POP to K-LAW. In XIV Seminario Internacional de investigación sobre la Cuenca del Pacífico (p. 11). Recuperado de: https://doi. org/10.13140/RG.2.1.2254.1201

- Ryzhkov, A., \& López Rocha, N. (2017). Hallyu y su percepción por los jóvenes coreanos en el contexto de la marca país coreana. Revista Digital Mundo Asia Pacífico, 6, 11, 6-26. Recuperado de: https://doi. org/10.17230/map

- Schulze, M. (2013). Korea vs. K-Dramaland: The Culturalization of K-Dramas by International Fans. Acta Koreana, 16(2), 367-397. Recuperado de: https://doi.org/10.18399/acta.2013.16.2.004

- Se-Woong, K. (29 de 8 de 2014). Korean Exposé. Obtenido de A Brief Note on the History of Korean Cannibalism. Recuperado de: https://www.koreaexpose.com/why-south-koreans-crave-human-flesh/

- $\quad$ Shim, D. (2011). Whither the Korean Film Industry? Acta Koreana, 14(1), 213-227.

- Smith, P. B. (2011). Cross-Cultural Perspectives on Identity. In S. J. S. et Al (Ed.), Handbook ofldentity Theory and Research (pp. 249-265). Sussex: Springer Science+Business Media. Recuperado de: https:// doi.org/10.1007/978-1-4419-7988-9_11

- Sung, S. Y. (2010). Constructing a new image. Hallyu in Taiwan. European Journal of East Asian Studies, 9(1), 25-45. Recuperado de: https://doi.org/10.1163/156805810X517652

- Valadez, C. A. (2016). Conceptualización de un segmento de mercado particular identificado con e K-pop en México. Portes, Revista Mexicana de Estudios Sobre La Cuenca Del Pacífico, 10(20), 181-201. Recuperado de: http://search.ebscohost.com/login.aspx?direct=truegdb=a9h\&AN=120376094\&lang $=$ es\&site $=$ ehost-live

- Yoon, S. (2015). Taming the Primitive: Multiculturalism and the Anthropological Vision of South Korean Media Visual Anthropology, 28(5), 422-437. Recuperado de: https://doi.org/10.1080/08949468.2015.1086209 